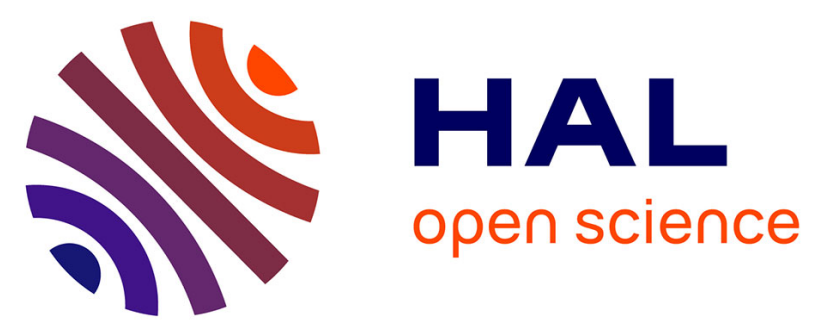

\title{
Interpretation of X-ray stress measurement and evaluation of internal residual stresses in rolled $\alpha$-Ti40 using self-consistent models
}

David Gloaguen, Manuel François, Ronald Guillen, Jean Royer

\section{- To cite this version:}

David Gloaguen, Manuel François, Ronald Guillen, Jean Royer. Interpretation of X-ray stress measurement and evaluation of internal residual stresses in rolled $\alpha$-Ti40 using self-consistent models. physica status solidi (a), 2002, 193 (1), pp.12-25. 10.1002/1521-396X(200209)193:13.0.CO;2-J . hal01006996

\section{HAL Id: hal-01006996 \\ https://hal.science/hal-01006996}

Submitted on 16 Jun 2014

HAL is a multi-disciplinary open access archive for the deposit and dissemination of scientific research documents, whether they are published or not. The documents may come from teaching and research institutions in France or abroad, or from public or private research centers.
L'archive ouverte pluridisciplinaire HAL, est destinée au dépôt et à la diffusion de documents scientifiques de niveau recherche, publiés ou non, émanant des établissements d'enseignement et de recherche français ou étrangers, des laboratoires publics ou privés.

\section{(c)(1)}

Distributed under a Creative Commons Attribution| 4.0 International License 


\title{
Interpretation of X-Ray Stress Measurement and Evaluation of Internal Residual Stresses in Rolled $\alpha$-Ti40 Using Self-Consistent Models
}

\author{
D. Gloaguen (a), M. Franc, ois (b), R. Guillen (a), and J. Royer (c) \\ (a) Laboratoire d'Applications des Matériaux à la Mécanique (LAMM), C.R.T.T., \\ Boulevard de l'Université, B.P. 406, 44602 Saint-Nazaire cedex, France \\ (b) Laboratoire des Systèmes Mécaniques et d'Ingénierie Simultanée (LASMIS), \\ Université de Technologie de Troyes, 11 rue Marie Curie, B.P. 2060, 10010 Troyes, France
}

(c) Laboratoire Mécanique et Matériaux (LMM), Ecole Centrale de Nantes, 1 rue de la Noë, B.P. 92101, 44321 Nantes cedex 03, France

\begin{abstract}
Internal stresses due to anisotropic thermal and plastic properties were investigated in rolled $\alpha$-titanium. The thermal stresses induced by a cooling process were predicted using a self-consistent model and compared with experimental results obtained by X-ray diffraction. A study of the elastoplastic response after uniaxial loading was performed along the rolling and the transverse direction of the sheet. Using an elastoplastic self-consistent model, the predicted results were compared with X-ray diffraction and mechanical tests. Theoretical and experimental results agree in their tendencies. The comparison between $\varepsilon_{\phi \psi}$ versus $\sin ^{2} \psi$ and simulations confirms that prismatic slip is the main active deformation mode.
\end{abstract}

\section{Introduction}

Titanium has a low density, a high resistance to corrosion, and a high strength-to-density ratio as well as other attractive mechanical properties. These properties have increased its use for chemical plant construction as well as in aerospace technology. Titanium alloys ( $\alpha$-phase) have a hexagonal close packed (hcp) structure at room temperature. Like most hcp metals, they are anisotropic in their properties at mesoscopic (grain) and macroscopic levels $[1,2]$. When the material is subjected to thermomechanical treatments, these properties and the crystallographic texture explain the appearance and the development of residual stresses that may be significant. It is essential to understand the mechanisms responsible for the creation and evolution of internal stresses because their influence on the mechanical performance of the material is generally important. The purpose of the present work is to combine experimental observations (X-ray diffraction, tensile tests) with the predictions of a self-consistent elastoplastic model in order to obtain more information about the different factors responsible for the appearance of residual elastic mesostrains. This comparison allows a better understanding and interpretation of X-ray diffraction and mechanical results. X-ray diffraction is one of the most often used techniques to characterise the mechanical state at the mesoscopic level of polycrystalline materials. 
In a previous paper [3], we have presented a study concerning the development and the evolution of residual stresses introduced by tensile tests in rolled textured polycrystalline $\alpha-Z r 702$ after a cooling process. It is interesting to extend this investigation to a rolled hexagonal material with $c / a$ close to the zirconium value (1.5873), $\alpha$-Ti40. It resembles zirconium in its chemical and physical properties. A self-consistent model of elastoplastic deformation was used to simulate the thermal and the tensile response of samples tested along the rolling and the transverse directions (RD and TD) of the sheet. Contrary to zirconium alloys, titanium is characterized by a low thermal anisotropy [4]. It seems interesting to quantify precisely the level and the influence of the thermal stresses on the macroscopic behaviour and to compare with zirconium. The problem of stress-free lattice parameter determination is also analysed using the selfconsistent model and experimental results obtained by X-ray diffraction. In this study, we attempt to understand the evolution of the mechanical residual stresses in textured titanium alloys when an elastoplastic deformation is introduced. The problem is studied using an elastoplastic self-consistent model. Predicted results for internal stresses introduced by tensile tests are compared with experimental data provided by X-ray diffraction experiments.

\section{Experimental Procedure}

The investigated material is commercially pure $\alpha$-titanium (Ti40). Its chemical composition is (wt\%-balance = Ti) C (0.07), $\mathrm{N}(0.008)$, O (0.12), and $\mathrm{H}$ (0.0013). The metallographic characterisation of the annealed material revealed an average grain size of approximately $40 \mu \mathrm{m}$.

\subsection{Mechanical testing}

To analyse the influence of loading direction and texture on the mechanical response of the material, samples of dimensions $2 \times 1.5 \times 43.4 \mathrm{~mm}^{3}$ were cut from the rolling and the transverse directions of the sheet. The specimens were submitted to uniaxial tensile

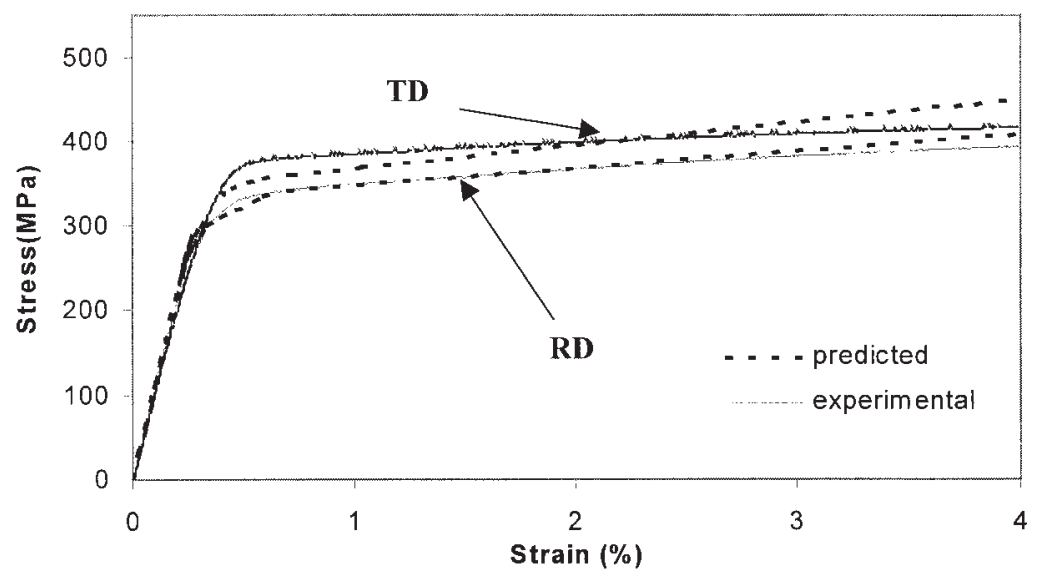

Fig. 1. Experimental and calculated stress-strain curves for a tensile test in the rolling and transverse directions of the sample 
tests at room temperature, at a constant strain rate of $3.8 \times 10^{-3} \mathrm{~s}^{-1}$ up to strains $1 \%$, $2 \%$, and $4 \%$ in the two loading directions. The tensile loading curves are depicted in Fig. 1.

\subsection{Stress measurements by the X-ray diffraction technique}

The experiments were carried out in two stages:

- Characterization of internal stresses due to the thermal anisotropy in an undeformed sample after a thermal treatment at $650{ }^{\circ} \mathrm{C}$ for $4.5 \mathrm{~h}$.

- Determination of the evolution of internal stresses due to the plastic anisotropy in deformed samples after uniaxial loading both along rolling and transverse directions.

These experiments were carried out on a D500 Siemens goniometer with $\mathrm{Cu}-\mathrm{K}_{\alpha}$ radiation. An $\Omega$ goniometric assembly with a scintillation detector was used. Two plane families were studied, $\{21 \overline{3} 3\}$ at $2 \theta=139.731^{\circ}$ and $\{30 \overline{3} 2\}$ at $2 \theta=148.751^{\circ}$. Diffractograms were recorded for fifteen tilt angles $\psi$ varying between $-45^{\circ}$ and $45^{\circ}$ and for azimuth angles $\phi=0^{\circ}, 45^{\circ}$, and $90^{\circ}$. The direction $\phi=0^{\circ}$ corresponds to the tensile direction. The X-ray beam output collimator has $0.5 \mathrm{~mm}$ diameter and a diffractedbeam slit limits the equatorial divergence to $0.6^{\circ}$. The peak position is determined by the half value breadth. The stress is obtained from the $\sin ^{2} \psi$ relation using the macroscopic elasticity constants. Because of the low mesoscopic elastic anisotropy (single crystal level), $\varepsilon_{\phi \psi}$ versus $\sin ^{2} \psi$ curves for a given family of diffracting planes $\{h k i l\}$ can be represented by straight lines. In this case, the X-ray elasticity constants (XEC) can be treated as those corresponding to an isotropic material. Texture analysis shows a high density of basal poles around the normal direction (ND), and prismatic poles are uniformly spread in the RD-TD and RD-ND planes. The initial texture is shown in Fig. 2.

To check stress homogeneity in the depth of the sample, the specimens were electrochemically polished. Electro-polishing was carried out at $298 \mathrm{~K}$ under a voltage of $33 \mathrm{~V}$ in a $10 \%$ perchloric acid and $90 \%$ acetic acid solution. Stress measurements by X-ray

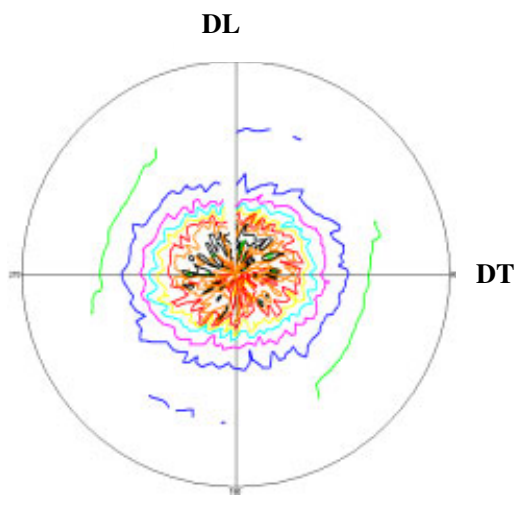

Max.: $2.62^{\circ}$

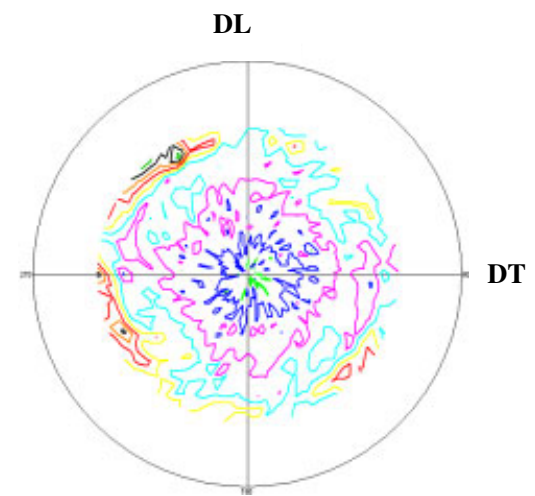

Max.: $12.63^{\circ}$

$\{0002\}$

Fig. 2. (online colour) Experimental texture of rolled Ti40, $\{0002\}$ and $\{10 \overline{1} 0\}$ pole figures 
diffraction were made at different depths with samples deformed along the RD at $1 \%$ and $2 \%$ total strain. The experimental data from the mechanical tests and X-ray diffraction are presented and compared with the results from our model in Section 4.

\section{Self-Consistent Modeling}

We assume that thermal contractions do not introduce plastic deformation in the material during the cooling process [4]. In this case, a thermoelastic formulation is sufficient to simulate the evolution of thermal stresses associated with cooling. An elastoplastic self-consistent scheme was then used to predict the mechanical behaviour of titanium polycrystal under an uniaxial tension.

The model used for our simulations was presented in a previous paper [3]. More details can be found in references [5-8]. The self consistent model is based on the following assumptions:

- Each grain is considered as a spherical inclusion embedded in a homogeneous effective medium (HEM) having the average properties of the aggregate.

- The mesoscopic stress $\boldsymbol{\sigma}$ and the mesoscopic strain $\varepsilon$ in the inclusion are homogeneous.

- The overall response of the material is determined by averaging the microscopic terms $(\sigma, \varepsilon)$ over a representative set of orientations.

- The macroscopic stress $\boldsymbol{\Sigma}$ and macroscopic strain $\mathbf{E}$ are considered as uniform. The properties of the medium are initially undetermined and are solved by iterations. Each grain is treated as an inhomogeneity with elastic $(c)$ and thermal $(\alpha)$ constants, slip and twin mechanisms characteristic of a single crystal.

- The texture is known, the polycrystal is represented by a weighted discrete distribution of orientations (Euler angles $\left(\varphi_{1} \varphi \varphi_{2}\right)$ ). Lattice rotation (reorientation by slip and twinning) and texture changes are incorporated in the model. However, as could be expected for such small strain $(<5 \%)$, the effect of texture change is negligible.

\subsection{Thermal residual stresses}

The thermal strains due to cooling from annealing temperature to room temperature are calculated with the thermoelastic model. It was assumed that the polycrystal is stress-free at $923 \mathrm{~K}$. The crystallographic orientation distribution function of the sample was calculated from the pole figures of the sample. A set of 1000 orientations was taken randomly and each orientation was given a volume fraction according to the texture function. The thermal and elastic properties of single-crystal titanium are listed in Table 1. The thermal strains due to a temperature variation from 923-298 K are calculated.

The thermal and elastic coefficients depend on temperature. The variation of $c_{i j}$ and $\alpha_{i j}$ with temperature was taken into account in the model through the measured values

Table 1

Thermal expansion coefficients (in $10^{-6} \mathrm{~K}^{-1}$ ) [4] and elastic constants (in GPa) [11] at single crystal level and at room temperature. Here $x_{3}$ is parallel to the $c$-axis

\begin{tabular}{lllllll}
\hline$\alpha_{11}\left(=\alpha_{22}\right)$ & $\alpha_{33}$ & $c_{11}$ & $c_{33}$ & $c_{44}$ & $c_{12}$ & $c_{13}$ \\
\hline 9.9 & 12.0 & 162.4 & 180.7 & 46.7 & 92.0 & 69.0 \\
\hline
\end{tabular}


Table 2

Coefficients of the polynomial relation that describes the temperature dependence of the elastic constants of titanium single crystals measured by Fisher and Renken [9]

\begin{tabular}{lcccc}
\hline coefficients & $c_{i j}^{(0)} \times 10^{0}$ & $c_{i j}^{(1)} \times 10^{3}$ & $c_{i j}^{(2)} \times 10^{6}$ & $c_{i j}^{(3)} \times 10^{9}$ \\
\hline$c_{11}$ & 177.421 & -44.199 & -26.166 & 19.287 \\
$c_{12}$ & 86.053 & 19.007 & 4.305 & -9.930 \\
$c_{13}$ & 0.679 & 0.047 & -0.041 & -0.003 \\
$c_{33}$ & 191.408 & -33.261 & -13.844 & 10.880 \\
$c_{44}$ & 51.105 & -10.329 & -15.860 & 7.202 \\
\hline
\end{tabular}

of Fisher and Renken [9] (elasticity) and Wasilewski [10] (thermal). They can be described by polynomial relations,

$$
\begin{aligned}
& c_{i j}=c_{i j}^{(0)}+c_{i j}^{(1)} T+c_{i j}^{(2)} T^{2}+c_{i j}^{(3)} T^{3}, \\
& \alpha_{i}=\alpha_{i}^{(0)}+\alpha_{i}^{(1)} T+\alpha_{i}^{(2)} T^{2} .
\end{aligned}
$$

The calculated coefficients $c_{i j}^{(n)}$ and $\alpha_{i}^{(n)}$ are listed, respectively, in Tables 2 and 3 .

\subsection{Mechanical residual stresses}

An incremental elastoplastic self-consistent scheme was used to simulate the mechanical loading. At each strain increment, the non-linear behaviour is approximated by the tangent behaviour. The stress and strain rates, $\dot{\boldsymbol{\sigma}}$ and $\dot{\boldsymbol{\varepsilon}}$, of each grain are linearly linked by an elastoplastic tensor $\mathbf{I}$. This tensor accounts for the plastic and elastic anisotropy of the grains and the hardening effects of the deformation systems during plasticity. The tensor $\mathbf{I}$ can be written as

$$
\mathbf{I}=\mathbf{c}-\sum_{h, g} \mathbf{c} . . \mathbf{R}^{h} \ldots\left(\mathbf{R}^{g} . . \mathbf{c} . . \mathbf{R}^{h}+\mathbf{H}^{g h}\right)^{-1} . . \mathbf{R}^{g} . . \mathbf{c},
$$

where $\mathbf{c}$ is the elastic stiffness of the single crystal, $\mathbf{H}$ the hardening matrix, $\mathbf{R}^{g}$ is the Schmid tensor of a system $g$, and ".." symbolizes the contracted tensor product. The summation is performed over all the active systems $h$ and $g$.

At the macroscopic level, the relation between the macroscopic stress rate and strain rate is given by a similar relation with the introduction of tensor $\mathbf{L}$. The values of $\dot{\boldsymbol{\sigma}}$ and $\dot{\boldsymbol{\varepsilon}}$ of each grain are linked to $\dot{\boldsymbol{\Sigma}}$ and $\dot{\mathbf{E}}$ by an interaction tensor $\mathbf{L}^{*}$ function of an Eshelby tensor [8] taking into account the intrinsic elastic and plastic anisotropy of the HEM as well as the anisotropy induced by plastic flow. (The tangent macroscopic operator $\mathbf{L}$ used to calculate $\mathbf{L}^{*}$ satisfies both normality and consistency conditions.) It is a function of $\mathbf{L}$, the shape and the orientation of each grain [5],

$$
\dot{\boldsymbol{\sigma}}-\dot{\boldsymbol{\Sigma}}=\mathbf{L} . .\left[\mathbf{I}-\mathbf{S}^{\mathrm{Esh}-1}\right] . .(\dot{\boldsymbol{\varepsilon}}-\dot{\mathbf{E}})=-\mathbf{L}^{*} . .(\dot{\boldsymbol{\varepsilon}}-\dot{\mathbf{E}}) .
$$

Table 3

Coefficients of the polynomial relation that describes the temperature dependence of the thermal coefficients of titanium single crystals measured by Wasilewski [10]

\begin{tabular}{lllll}
\hline coefficients & $\alpha_{i}^{(0)} \times 10^{6}$ & $\alpha_{i}^{(1)} \times 10^{9}$ & $\alpha_{i}^{(2)} \times 10^{12}$ & $\alpha_{i}^{(3)} \times 10^{15}$ \\
\hline$\alpha_{1}$ & 12.791 & 8.110 & -37.442 & 30.379 \\
$\alpha_{3}$ & 12.088 & 24.603 & -74.074 & 50.252 \\
\hline
\end{tabular}


The overall elastoplastic tensor $\mathbf{L}$ is a weighted average of the mesoscopic tensor $\mathbf{l}$,

$$
\mathbf{L}=\left\langle\mathbf{I} . .\left[\mathbf{I}+\mathbf{S}^{\text {Esh }} . . \mathbf{L}^{-1} . . \Delta \mathbf{I}\right]^{-1}\right\rangle .
$$

Equation (4) is a non-linear implicit equation because $\mathbf{S}^{\text {Esh }}$ depends on the unknown $\mathbf{L}$. The Eshelby tensor is calculated by an integral equation [7] which takes into account plastic anisotropy. Equation (4) is solved by iteration. Once $\mathbf{L}$ is known, by specifying an overall stress or strain, the model can give the corresponding stress or strain tensors for each orientation. Thus, we can describe the mechanical response of the polycrystal at the mesoscopic scale.

For hexagonal materials like titanium, because of the relatively strong crystallographic anisotropy, several modes may be active: slip and/or twinning systems. In such materials, prismatic glide $\{10 \overline{1} 0\}\langle 11 \overline{2} 0\rangle$ is reported to be the main active deformation mode [2, 12]. First-order pyramidal slip $\{10 \overline{1} 1\}\langle 11 \overline{2} 3\rangle$ and basal slip $\{0002\}\langle 11 \overline{2} 0\rangle$ are generally presented as secondary slip modes $[2,13,14]$. At room temperature, twinning has also been observed in titanium samples [14, 15]. Twinning of $\{10 \overline{1} 2\}$ and $\{11 \overline{2} 1\}$ types is expected in extension along the $c$-axis whereas twinning of $\{11 \overline{2} 2\}$ and $\{10 \overline{1} 1\}$ types is expected in compression along the $c$-axis.

In the model, critical resolved shear stresses (CRSS) and the hardening law for slip and twinning are considered as controlling parameters. As their values depend on many factors (oxygen content, grain size, temperature,...), they are considered as unknown. Consequently, they are determined through a fitting of the predicted behaviour on the experimental tensile curve in RD.

The initial CRSS are taken as identical for all systems of a system type. The deformation systems introduced in the model are assumed to be prismatic slip, first-order pyramidal slip, and $\{10 \overline{1} 2\}$ twinning. In this work, we considered a linear hardening law [16], and for simplicity, coefficient $H^{g r}$ is equal to $H^{g g}$ for any deformation mode $r: \dot{\tau}^{g}=H^{g} \sum_{r} \dot{\gamma}^{r}$, i.e. latent hardening is equal to self-hardening. The CRSS obtained are $\tau=120 \mathrm{MPa}$ for prismatic mode, $\tau=190 \mathrm{MPa}$ for first-order pyramidal mode, and $\tau=210 \mathrm{MPa}$ for tensile twinning. The hardening coefficients were found to be $40 \mathrm{MPa}$ for prismatic slip, $80 \mathrm{MPa}$ for pyramidal slip, and $210 \mathrm{MPa}$ for the twin mode. The CRSS for the prismatic systems is determined to reproduce the experimental yield stress in the RD. The CRSS values are adjusted to give a good description of the tensile test performed in the RD of the sheet. Experimental observations [15] show that twinning does not make a significant contribution to plastic deformation. A CRSS value of $210 \mathrm{MPa}$ ensures that twinning activity is lower than prismatic or pyramidal activity. The hardening coefficients are chosen to be consistent with the slope of the experimental curve in the RD of the sample. The obtained parameters are then taken to simulate the tensile test in the TD. Two assumptions were considered in the simulations: loading with thermal stresses and without thermal stresses.

\section{Results and Comparison between Model and Experiment}

\subsection{Thermal stresses}

The evolution of thermal stresses as a function of temperature was predicted for different plane families,

$-\{21 \overline{3} 3\}$ and $\{30 \overline{3} 2\}$, which are the planes studied by X-ray diffraction, 


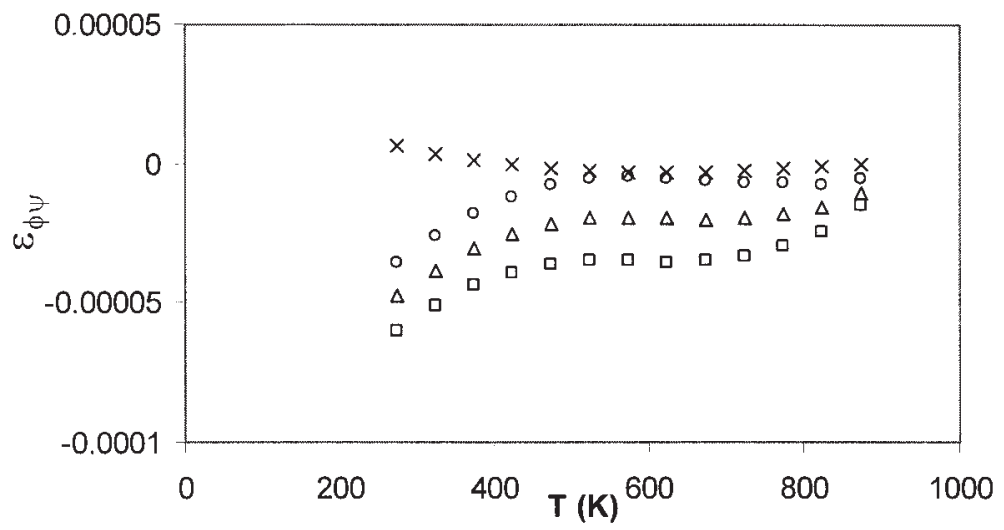

Fig. 3. Predicted thermal strains for the different planes studied with $\phi=0^{\circ}$ and $\psi=0^{\circ}(\square\{10 \overline{1} 0\}$, $o\{21 \overline{3} 3\}, \Delta\{30 \overline{3} 2\}, \times\{0002\})$

- $\{0002\}$ and $\{10 \overline{1} 0\}$ are, respectively, the basal and the prismatic planes. These planes are interesting to study because they generally have an opposite behaviour in terms of strain values.

Figure 3 shows the residual stress evolution obtained using the thermo-elastic selfconsistent method. Prismatic planes are in a compressive strain state while basal planes are in tensile strain state. These results are similar to previous simulations and experimental results obtained on rolled Zircaloy- 2 and $\alpha-\operatorname{Zr}[2,17]$. The directions normal to $\{21 \overline{3} 3\}$ and $\{30 \overline{3} 2\}$ planes are in compression.

To give an idea of the stress magnitude, the diagonal components $\sigma_{22}$ and $\sigma_{33}$ of the stress tensor can be calculated for every grain. These values are expressed in crystal axis (with $x_{3}$ parallel to the $c$-axis) and correspond to values obtained after cooling at room temperature. Contrary to zirconium alloys, these values are low: the $\sigma_{33}$ values are always less than $6 \mathrm{MPa}$ and the $\sigma_{22}$ values are higher than $-7 \mathrm{MPa}$.

Using the self-consistent formulation, thermal residual strains projected on the measurement direction versus $\sin ^{2} \psi$ for the $\{21 \overline{3} 3\}$ and $\{30 \overline{3} 2\}$ diffracting planes were simulated. These strains can be represented by a straight line $A+B \sin ^{2} \psi$. The predicted results are presented in Table 4.

Physically, the values of $A$ must be independent of $\phi$. This fact allows to estimate the numeric precision of the calculations. We can express the values $A$ in terms of stress with the XEC (determined by an elastic self-consistent model for an isotropic material). The standard deviation is of the order of $0.2 \mathrm{MPa}$ for the two studied planes. The choice of 1000 orientations is justified by this low dispersion.

Table 4

Predicted coefficients $A$ and $B$ for the $\{21 \overline{3} 3\}$ and $\{30 \overline{3} 2\}$ diffracting planes

\begin{tabular}{|c|c|c|c|c|c|c|}
\hline & \multicolumn{3}{|c|}{$21 \overline{3} 3$} planes & \multicolumn{3}{|c|}{$30 \overline{3} 2$} planes \\
\hline & $\phi=0^{\circ}$ & $\phi=45^{\circ}$ & $\phi=90^{\circ}$ & $\phi=0^{\circ}$ & $\phi=45^{\circ}$ & $\phi=90^{\circ}$ \\
\hline$A\left(\times 10^{3}\right)$ & -0.04356 & -0.04304 & -0.04305 & -0.05621 & -0.05846 & -0.05826 \\
\hline $\begin{array}{l}B\left(\times 10^{3}\right) \\
A / \mathrm{XEC}(\mathrm{MPa})\end{array}$ & $\begin{array}{l}0.05957 \\
15.5\end{array}$ & $\begin{array}{l}0.05603 \\
15.3\end{array}$ & $\begin{array}{l}0.05766 \\
15.3\end{array}$ & $\begin{array}{l}0.06270 \\
20.0\end{array}$ & $\begin{array}{l}0.06291 \\
20.8\end{array}$ & $\begin{array}{l}0.06 \\
20.7\end{array}$ \\
\hline
\end{tabular}


The predicted results are compared with experimental data obtained using the X-ray diffraction method. The experimental strain values are determined using the expression

$$
\varepsilon_{\phi \psi}=-\frac{1}{2} \cot \theta_{0}\left(2 \theta_{\phi \psi}-2 \theta_{0}\right)
$$

where $\theta_{0}$ is the Bragg angle for a stress-free specimen and $\theta_{\phi \psi}$ is the measured Bragg angle. Consequently, it is necessary to determine the stress-free lattice parameters of the sample to calculate the thermal strains. Because of strains induced by thermal anisotropy, these parameters cannot be directly measured. One solution [18] is to find the stress-free parameters $a_{0}$ and $c_{0}$ which minimize the difference between experimental data and predicted strains for the two studied planes. With this method, the following values were found: $a=2.9521 \AA$ and $c=4.6893 \AA$, giving $2 \theta_{0}(\{21 \overline{3} 3\})=139.162^{\circ}$ and $2 \theta_{0}(\{30 \overline{3} 2\})=148.194^{\circ}$.

Figure 4 shows the experimental (filled circles and triangles) and predicted (full line) thermal strains versus $\sin ^{2} \psi$ curves for $\{21 \overline{3} 3\}$ and $\{30 \overline{3} 2\}$ diffracting planes with

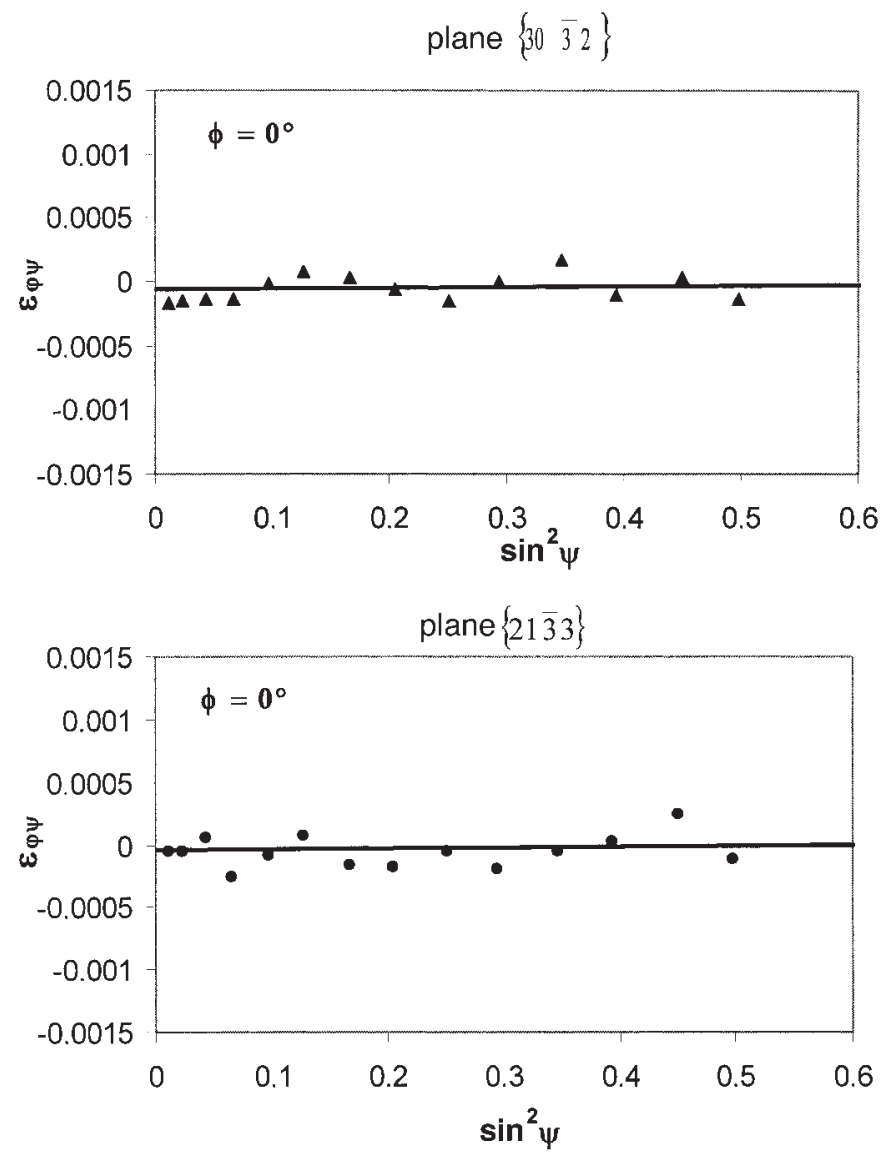

Fig. 4. Thermal residual strains vs. $\sin ^{2} \psi$ for the $\{30 \overline{3} 2\}$ and $\{21 \overline{3} 3\}$ diffracting planes at room temperature with $\phi=0^{\circ}(\bullet\{21 \overline{3} 3\}$ experimental results, $\Delta\{3032\}$ experimental results, solid line predicted results obtained using the self-consistent model) 
$\phi=0^{\circ}$. The experimental dispersion can be attributed to grain size effects and counting statistics. It can be noticed that strain (or stress) values are very weak compared to the case of zirconium alloys [3]. The slope of $\varepsilon_{\phi \psi}$ versus $\sin ^{2} \psi$ is close to 0 .

In conclusion, thermal stresses at room temperature are weak and do not play, a priori, an important role for the elastoplastic behaviour (see next section). Using the self-consistent model and the experimental results from X-ray diffraction, the stress-free lattice parameters of the sample can be determined. Although the thermal anisotropy of titanium is weak, a (too) simple comparison of bulk thermal strain and crystal thermal strain along the $c$-axis would give stresses of the order of $100 \mathrm{MPa}$ with the relation

$$
\sigma=E\left(M_{11}-\alpha_{33}\right) \Delta T
$$

where $E$ is the Young's modulus and $M_{11}\left(\cong M_{22} \cong M_{33}\right)$ is the thermal expansion coefficient of the polycrystal in the RD.

The model calculations and the experimental results show that the effective thermal stresses are low due to elastic coupling at the crystallite scale.

\subsection{Mechanical response}

Figure 1 shows the predicted stress-strain curves for a tensile test in rolling and transverse directions. The simulation is fitted on the experimental loading curve along the $\mathrm{RD}$ of the sheet. The predicted yield stress is about $251 \mathrm{MPa}(0.23 \%$ total strain). This value is very close to the experimental one $(253 \mathrm{MPa})$. The calculated Young's modulus $(107 \mathrm{GPa})$ coincides with the experimental one. The predicted and measured plastic slopes are identical up to 3\% strain along the RD. After 3\% strain, the tensile curve saturates. This effect cannot be reproduced by the model with simple constant hardening coefficients. A more sophisticated phenomenological hardening model would be necessary. With a loading along the TD, the initiation of the elastoplastic transition begins at $271 \mathrm{MPa}(0.27 \%$ strain). The experimental yield stress is about $293 \mathrm{MPa}$. Although the predicted yield stress is lower than the experimental one, the simulation matches the fact that the yield stress in TD is higher than in RD. The simulated plastic slope $(2800 \mathrm{MPa})$ is significantly higher than the experimental one $(1250 \mathrm{MPa})$. This fact could be attributed to variations of CRSS and hardening coefficients within grains and for different systems of the same system type. However, our results show that the crystallographic texture can explain an important part of the anisotropic behaviour of the material. The remaining can be attributed to the morphological anisotropy or the intracrystalline anisotropy which is not fully taken into account in the hardening matrix. The simple model of hardening used here does not capture some macroscopic effects related to these mechanisms. More elaborated treatments of hardening (latent hardening, dislocation annihilation,...) could be introduced into the model to reach a better agreement with experimental observations.

For a tensile test in the RD, the elastoplastic transition starts with the activation of prismatic slip. At $0.7 \%$ strain, pyramidal slip is activated. This sequence explains correctly the experimental loading slope. At $4 \%$ deformation, the proportions of activated systems are $61 \%$ for prismatic mode and $39 \%$ for pyramidal slips. The twinning is not active during the deformation with our choice of CRSS.

When tension is simulated in the TD, plastic deformation begins by the activation of prismatic slip. At $0.5 \%$ strain, pyramidal systems become active. At $4 \%$, the distribu- 
tion of activated systems is $70 \%, 28.9 \%$, and $1.1 \%$ for the prismatic, pyramidal, and tensile twin, respectively.

Finally, a reasonable distribution was obtained in agreement with the published experimental observations: prismatic glide is the main active mechanism, pyramidal slip is a secondary slip mode, and twinning contributes very little to plastic deformation. To quantify the influence of thermal stresses on the macroscopic response, calculations were also performed without thermal stress in the initial conditions. The predicted stress-strain curves are identical to the previous case. The elastoplastic transition begins at $256 \mathrm{MPa}$ (251 MPa with thermal stresses) in the RD. Pyramidal slip and twinning become active at the same strain. Thermal anisotropy is very low and its influence on the macroscopic behaviour is negligible.

\subsection{X-ray diffraction results}

Strain measurements have been made with two plane families, $\{21 \overline{3} 3\}$ and $\{30 \overline{3} 2\}$. The stress was obtained from the $\sin ^{2} \psi$ relation. In the present section, it should be remembered that, due to the homogeneous macroscopic plastic flow, there is no macroscopic
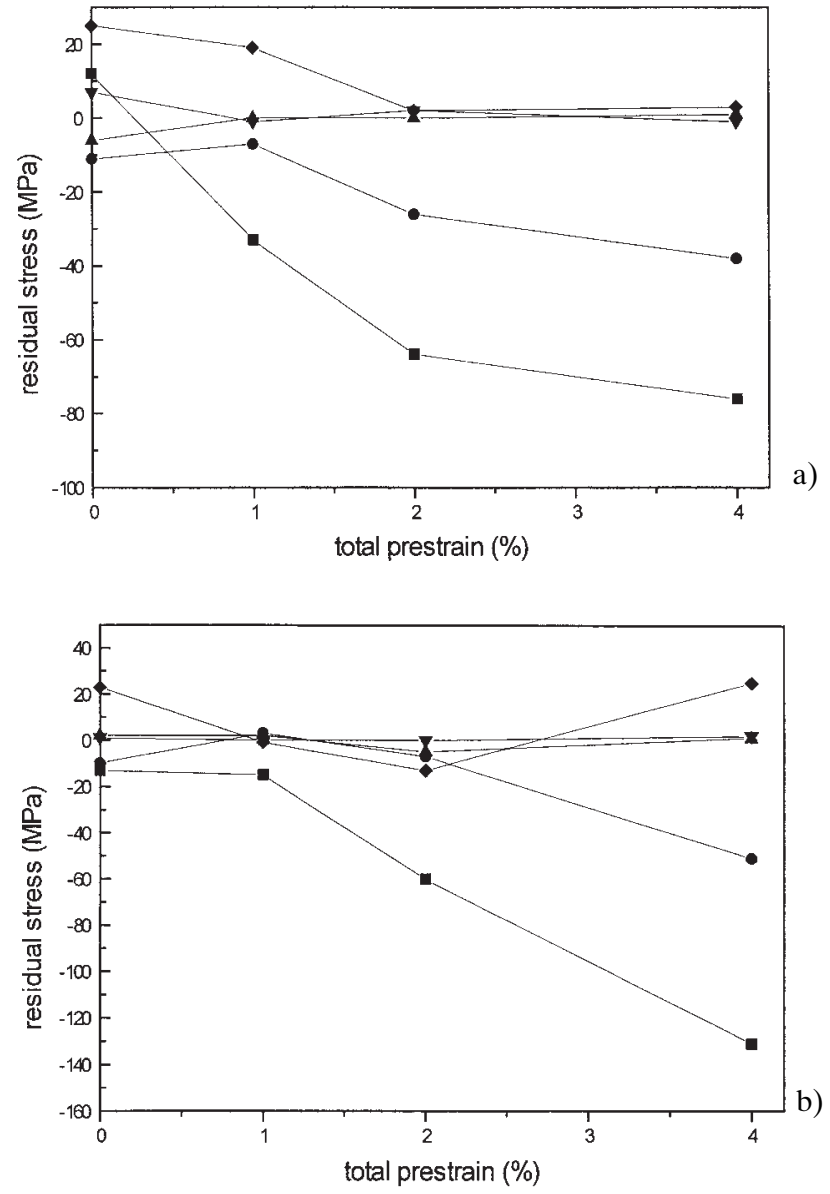

a)

Fig. 5. Evolution of residual stress tensor components with the total prestrain for the a) $\{21 \overline{3} 3\}$ and b) $\{30 \overline{3} 2\}$ planes with loading along RD. The $x_{1}$-axis is along the tensile direction (RD) ( $\sigma_{11}$, 
residual stress. The measured and simulated stresses are relative to the diffracting volume, i.e. to groups of crystallites with specific orientation. The mentioned stresses are thus pseudo-macroscopic stresses. With diffraction data, the complete stress tensor was determined for each plane family. To check the stress homogeneity in the sample, X-ray analysis has been performed with different thicknesses removed by electro-polishing. At $2 \%$ strain, pseudo-macroscopic stress varies from $-58 \pm 33 \mathrm{MPa}$ to $-49 \pm 18 \mathrm{MPa}$ for the $\{21 \overline{3} 3\}$ plane. Using the $\{30 \overline{3} 2\}$ reflection, the calculated stress varies between $-22 \pm 55 \mathrm{MPa}$ at the surface and $-49 \pm 23 \mathrm{MPa}$ at $125 \mu \mathrm{m}$ depth. Finally, stress analysis by X-ray diffraction shows that the stress seems to be homogeneous inside the samples. As the samples are single phased and the penetration depth of X-rays is very small $(5 \mu \mathrm{m})$, it was assumed that $\sigma_{33}=0$ to calculate the stress tensor. It is interesting to notice that, although the crystallites analysed by XRD are different when $\phi$ and $\psi$ angles vary, the whole set of data is consistent with a global stress tensor whose principal axes are very close to RD and TD. This can be explained by the fact that mesoscopic stress depends directly on the macroscopic stress and that the whole set of crystallites analysed by XRD is representative of a global incompatibility between the grains. This justifies the idea of pseudo-macroscopic stress. Figures 5 and 6 show the
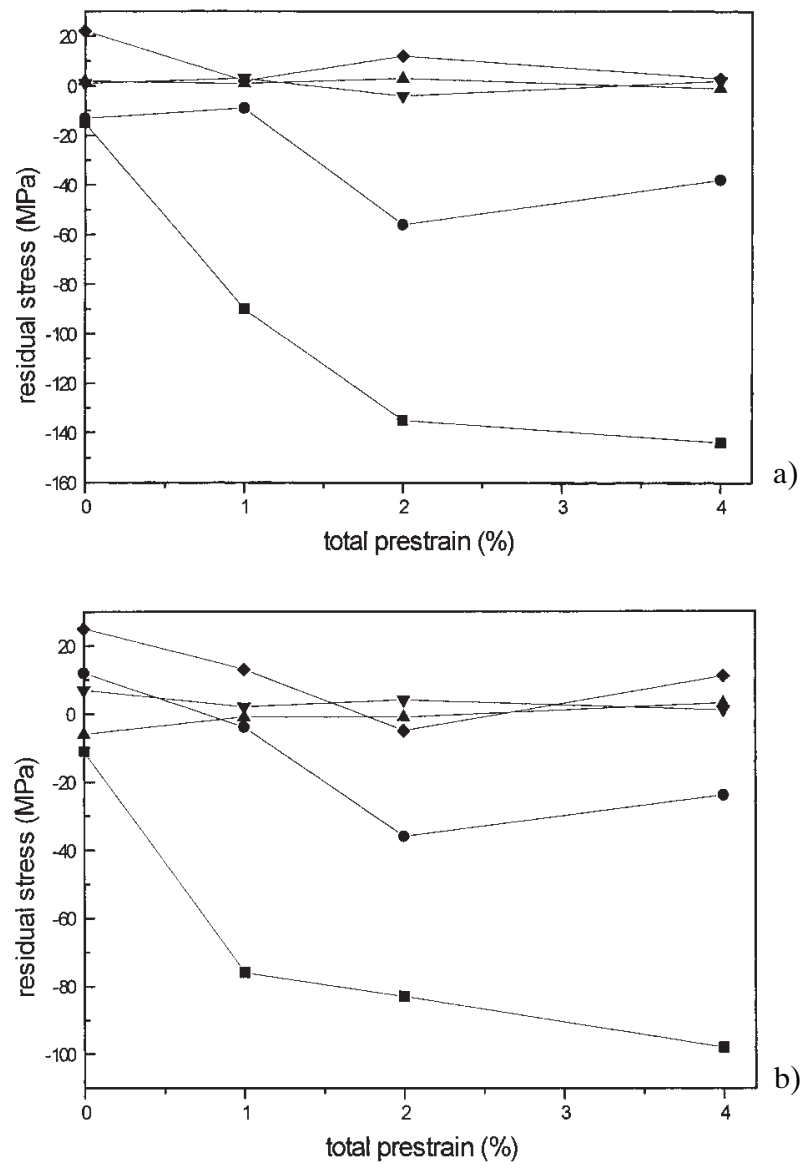

a)

Fig. 6. Evolution of residual stress tensor components with the total prestrain for the a) $\{21 \overline{3} 3\}$ and b) $\{3032\}$ planes with loading along TD. The $x_{1}$-axis is along the tensile direction $(\mathrm{RD})\left(\boldsymbol{\square} \sigma_{11}, \bullet \sigma_{22}\right.$, $\left.\Delta \sigma_{23}, \boldsymbol{\nabla} \sigma_{13}, \diamond \sigma_{12}\right)$ 


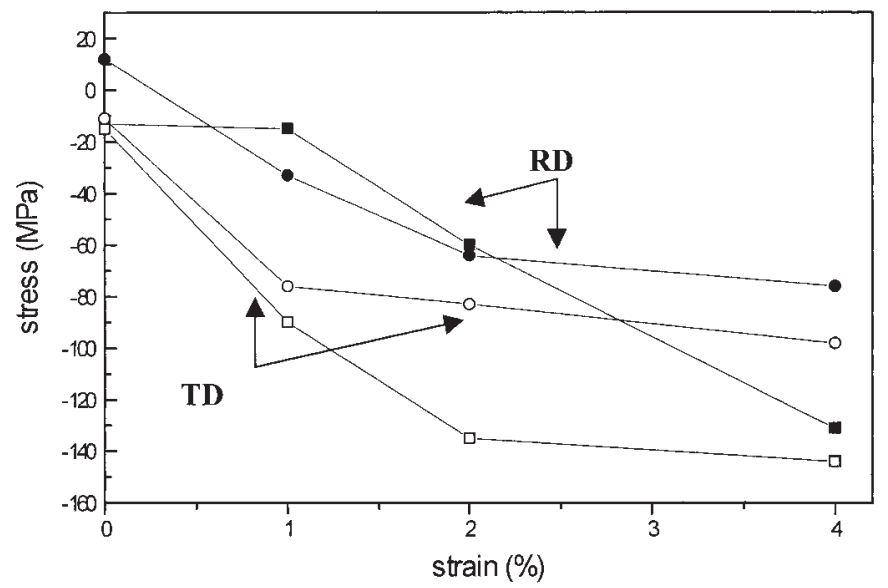

Fig. 7. Evolution of $\sigma_{11}$ with the total strain for $\{30 \overline{3} 2\}$ (circles) and $\{21 \overline{3} 3\}$ (squares) with loading along RD (filled symbols) and TD (open symbols). The $x_{1}$-axis is parallel to the loading direction (RD or TD)

evolution of components of the stress tensor as a function of the total strain with a loading along the RD and TD, respectively (the $x_{1}$-axis is taken parallel to the loading direction). The shear stress components remain close to $0 \mathrm{MPa}$ for any deformation. The two planes are in compression with a stress value that increases with the amount of prestrain. At $4 \%$ strain, the $\sigma_{11}$ value reaches $-140 \mathrm{MPa}$ for the $\{21 \overline{3} 3\}$ plane and $-80 \mathrm{MPa}$ for the $\{30 \overline{3} 2\}$ plane, while the $\sigma_{22}$ value is of the order of $-40 \mathrm{MPa}$. For the mechanical tests along the TD, stress values are also in compression along the rolling $\left(\sigma_{11}\right)$ and the transverse $\left(\sigma_{22}\right)$ direction for the two planes. The $\sigma_{11}$ component reaches $-144 \mathrm{MPa}$ for the $\{21 \overline{3} 3\}$ plane and $-98 \mathrm{MPa}$ for the $\{30 \overline{3} 2\}$ plane. The $\sigma_{22}$ values are, respectively, -38 and $-24 \mathrm{MPa}$. The shear stresses have a very weak value whatever the deformation. Figure 7 presents the evolution of $\sigma_{11}$ with uniaxial loading along the $\mathrm{RD}$ and TD. This component decreases more rapidly with a loading in TD. This behaviour could be attributed to the higher yield stress in the transverse direction. X-ray measurements show the existence of plastic anisotropy through two phenomena:

- a significant difference for the $\sigma_{11}$ value ( -144 and $-98 \mathrm{MPa}$ at $4 \%$ strain) between the two loading directions,

- a different evolution of $\sigma_{11}$ for the $\{21 \overline{3} 3\}$ plane $(-140 \mathrm{MPa})$ and the $\{30 \overline{3} 2\}$ plane $(-80 \mathrm{MPa})$.

For comparison, in cold rolled zircaloy-4 cladding tubes [19, 20], the stresses obtained by XRD depend strongly on the analysed plane family $(+168 \mathrm{MPa}$ for the $\{10 \overline{1} 4\}$ plane and $-193 \mathrm{MPa}$ for the $\{10 \overline{1} 1\}$ plane). This significant difference is not observed for the two studied planes in the case of titanium. However, it should be noted that these two planes are close in terms of crystallographic orientation, contrary to the $\{10 \overline{1} 4\}$ and $\{10 \overline{1} 1\}$ planes.

\subsection{Predicted mesoscopic strains}

The study in Section 4.2 enabled us to check the capacity of our model to simulate the macroscopic behaviour in rolled $\alpha$-Ti. The next step will be to validate the assumptions 


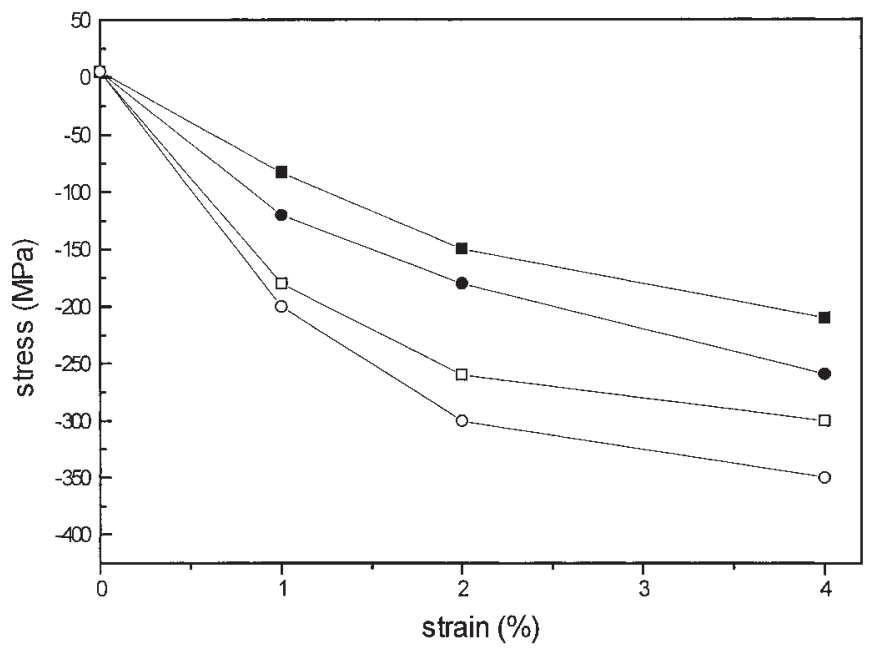

Fig. 8. Predicted evolution of $\sigma_{11}$ with the total strain for $\{30 \overline{3} 2\}$ (circles) and $\{21 \overline{3} 3\}$ (squares) with loading along RD (filled symbols) and TD (open symbols). The $x_{1}$-axis is parallel to the loading direction (RD or TD)

on the deformation modes activated in our calculation. For that, a comparison between stress measurements with diffraction techniques on these samples and simulation at the mesoscopic level has been achieved.

Experimental results obtained with XRD show that the $\{21 \overline{3} 3\}$ and $\{30 \overline{3} 2\}$ planes exhibit compression stress for all directions $\phi\left(0^{\circ}, 45^{\circ}\right.$, and $\left.90^{\circ}\right)$. The predicted results agree with the fact that, in the $\varepsilon_{\phi \psi}$ versus $\sin ^{2} \psi$ representation of the deformed samples, the best fitting for the two studied planes is obtained with prismatic slip as the main deformation mode. In fact, simulations using different combinations of plastic modes (pyramidal or basal slip as main active deformation mode) were made and it was found that only the prismatic system can explain the experimental behaviour. From the slope of the $\varepsilon_{\phi \psi}$ curves, it is possible to estimate the pseudo-macroscopic residual stresses as a function of total strain for the two loading directions. Figure 8 presents this evolution. The predicted and experimental results show that stresses evolve more rapidly from $0 \%-2 \%$ strain and more slowly beyond $2 \%$. Stress values are close for the two studied planes. With prismatic slip as main deformation mode, the model explains the fact that pseudo-macroscopic residual stresses are higher in the RD than TD. Although the predictions are in qualitative agreement with experimental evidence, the residual stresses are overpredicted by the self-consistent model. This is a well-known feature of this model [21], which is too "stiff" because it does not take into account the fluctuations of moduli in the matrix and the inclusion due to the nonlinearity of the mechanical behaviour [22-23]. In our case, the model overestimates the observed residual stress by at least a factor two.

\section{Conclusions}

In this study, the thermal residual stresses in rolled $\alpha$-Ti have been evaluated using a self-consistent model and compared with X-ray diffraction results. A good agreement 
between the two methods has been obtained. The residual stresses due to thermal anisotropy are low, and consequently they do not play a role for the elastoplastic transition, contrary to zirconium alloys. The stress-free lattice parameters of the samples were determined with the model, and we found $a=2.9521 \AA$ and $c=4.6893 \AA$.

An elastoplastic self-consistent approach has been developed in order to model the mechanical response of rolled Ti under uniaxial loading. We showed that the crystallographic texture can explain the stress differential between RD and TD of the sample and that the elastoplastic transition can be explained by texture and internal stresses. The choice of prismatic slip as principal deformation mode can explain the negative pseudo-macrostress values for the two studied planes for all $\phi$ directions $\left(0^{\circ}, 45^{\circ}\right.$, and $90^{\circ}$ ). The model allows to describe the rapid evolution of pseudo-macrostresses during the elastoplastic transition and the slow evolution after $2 \%$. It reproduces the fact that the pseudo-macrostresses are higher for loading along TD than along RD. These predicted results can be only obtained for a single set of slip modes and hardening parameters. This analysis shows that X-ray diffraction stress analysis could constitute an effective validation or identification of a model. Compared with microstructural observations such as those performed by TEM or EBSD, which are often limited because of strain hardening (especially for measurements on materials strained at $40 \%$ or $70 \%$ ), XRD may provide an alternative way to investigate the deformation mechanisms of textured polycrystals. In any case, a complete understanding of the deformation mechanisms of hexagonal materials will require various techniques, among which XRD can play a useful role.

\section{References}

[1] E. Tenckhoff, Deformation Mechanisms, Texture, and Anisotropy in Zirconium and Zircaloy, STP 966, 1916 Race Street, Philadelphia, PA 19103 (1988).

[2] P.G. PATRIDGe, Metall. Rev. 12, 169 (1967).

[3] D. Gloaguen, M. François, R. Guillen, and J. Royer, Acta Mater. (UK) 50, 871 (2001).

[4] M. Ortiz and A.A. Pochetrino, 4th European Conf. Residual Stresses Cluny (France), June 4-6, 1996 (p. 971) (Vol. 2).

[5] P.A. Turner, N. Christodoulou, and C.N. Tomé, Int. J. Plast. 11, 251 (1995).

[6] R. Hill, J. Mech. Phys. Solids 13, 89 (1965).

[7] P. LiPINSKI and M. BERVEILLeR, Int. J. Plast. 5, 149 (1989).

[8] U.F. Kocks, C.N. Tomé, and H.R. Wenk, Texture and Anisotropy, Cambridge University Press, Cambridge 1998.

[9] E.S. Fisher and C. Renken, Phys. Rev. 135, 484 (1964).

[10] R.J. WASILEWSKI, Trans. Metall. Soc. AIME 221, 1231 (1961).

[11] G. Simmons and H. Wang, Single Crystal Elastic Constants and Calculated Aggregate Properties, MIT Press, Cambridge (MA) 1971.

[12] A.A. Pochettino, N. Gannio, C. Vial Edwards, and R. Penelle, Scr. Metall. 27, 1859 (1992).

[13] X.L. TAN and H.C. Gu, Mater. Sci. Eng. 189, 77 (1994).

[14] A. AKhtar, Metall. Trans. A 6, 1105 (1975).

[15] D.R. Chichili, K.T. RAmesh, and K.J. Hemker, Acta Mater. (UK) 46, 1025 (1998).

[16] J.W.L. Pang, T.M. Holden, P.A. Turner, and T.E. Masson, Acta Mater. (UK) 47, 37 (1999).

[17] S.R. MacEwen, C. Tomé, and J. Faber, Acta Mater. (UK) 37, 979 (1989).

[18] M. Ortiz and A.A. Pochettino, J. Nucl. Mater. 229, 65 (1996).

[19] E. GIRARD, Doctoral thesis, University of Nantes, Nantes (France) 1993.

[20] R. Guillen, C. Cossu, M. François, and E. Girard, J. Nucl. Mater. 255, 174 (1998).

[21] J. KRIER, Doctoral thesis, University of Metz, Metz (France) 1993.

[22] A. Zaoui and R. Masson, Mater. Sci. Eng. A 285, 418 (2000).

[23] R. Masson, M. Bornert, F. Suquet, and A. Zaoui, J. Mech. Phys. Solids 48, 1203 (2000). 